Relations industrielles

Industrial Relations

\title{
Operations Research for Managerial Decisions, by Donald R. Plane and Gary A. Kochenberger, Homewood, Ill., Richard D. Irwin Inc., 1972, 321 pp.
}

\section{Jean-Louis Langevin}

Volume 28, numéro 3, 1973

URI : https://id.erudit.org/iderudit/028437ar

DOI : https://doi.org/10.7202/028437ar

Aller au sommaire du numéro

Éditeur(s)

Département des relations industrielles de l'Université Laval

ISSN

0034-379X (imprimé)

1703-8138 (numérique)

Découvrir la revue

Citer ce compte rendu

Langevin, J.-L. (1973). Compte rendu de [Operations Research for Managerial Decisions, by Donald R. Plane and Gary A. Kochenberger, Homewood, Ill., Richard D. Irwin Inc., 1972, 321 pp.] Relations industrielles / Industrial Relations, 28(3), 675-675. https://doi.org/10.7202/028437ar

Tous droits réservés @ Département des relations industrielles de l'Université Laval, 1973
Ce document est protégé par la loi sur le droit d'auteur. L’utilisation des services d'Érudit (y compris la reproduction) est assujettie à sa politique d'utilisation que vous pouvez consulter en ligne.

https://apropos.erudit.org/fr/usagers/politique-dutilisation/ 
intellectuelle des jeunes comme objectif, le manuel, tel quel, n'a pas une très grande valeur. Il ne peut être utilisé à cette fin qu'à la condition que le cadre conceptuel de fond et le contenu soient présentés aux étudiants d'une manière critique. Si cette condition est remplie, il peut être un bon manuel.

\section{Jean-Marie RAINVILLE}

Université de Montréal

Operations Research for Managerial Decisions, by Donald R. Plane and Gary A. Kochenberger, Homewood, Ill., Richard D. Irwin Inc., 1972, $321 \mathrm{pp}$.

L'administrateur doit prendre des décisions face à des situations, à des problèmes souvent complexes par le nombre de paramètres qu'ils contiennent, de sorte qu'une solution intuitive comporte de grands risques.

La recherche opérationnelle, c'est l'application de l'analyse quantitative aux problèmes des décisions administratives. C'est la mathématique au service de la prise de décision. L'analyste ou le «spécialiste » de la recherche opérationnelle peut aider le dirigeant à systématiser l'analyse d'un problème et la prise de décision. Il vient épauler, à l'aide d'un outil de rationalisation, le dirigeant qui doit prendre une décision. C'est ce dernier cependant qui toujours prend la décision et non l'analyste qui intervient que pour mieux éclairer le dirigeant.

J'ose espérer que l'intention des auteurs n'est pas de remettre en doute la nature de cette relation lorsqu'ils écrivent qu'ils ne voient pas de distinction significative entre la science du management et la recherche opérationnelle.

Ecrit pour des non-mathématiciens qui désirent plus qu'une connaissance de surface ou des « recettes de l'analyse quantitative, cet ouvrage se veut être avant tout une discussion du sens commun et des possibilités d'utilisation des nombres en vue d'aider le dirigeant faisant face à des problèmes décisionnels.

Même s'il s'adresse à des «profanes », Operations Research for Managerial Decisions utilise quantité de formules mathématiques. Cependant, avant d'utiliser des concepts tels que le calcul différentiel et les probabilités, les auteurs prennent soin d'en présenter auparavant, les concepts de base.

Plus qu'une simple présentation théorique de la recherche opérationnelle, l'ouvrage à le souci du lecteur en ce sens qu'il cherche à développer, chez le lecteur, une certaine habileté dans la formulation des problèmes en termes de modèles mathématiques. Pour favoriser ce transfert, les auteurs mettent, tout au long du bouquin, l'emphase sur les techniques les plus utilisées dans leur application aux problèmes administratifs. Les exemples abondants et les problèmes qu'on retrouve à la fin de chacun des chapitres favorisent cet apprentissage.

$\mathrm{Au}$ plan du contenu, la programmation linéaire - terme utilisé pour décrire un grand nombre de techniques visant à optimiser ou trouver la meilleure valeur d'une fonction (objective function), en tenant compte des contraintes ou des exigences - retient l'attention principale des auteurs qui, auparavant, ont pris soin de nous instruire sur la nature de la recherche opérationnelle et d'esquisser les éléments principaux d'une théorie de la décision. Le dernier chapitre (chap. 9) du livre est consacré à la simulation comme moyen, de plus en plus intéressant à mesure que croît la complexité des systèmes, pour analyser les problèmes décisionnels.

En résumé, cet ouvrage convient à tout administrateur qui désire prendre un contact « concret» avec un outil d'analyse des problèmes impliquant une décision.

\section{Jean-Louis LANGEVIN}

\section{Université Laval}

Atlas de l'emploi : Ville et île de Montréal, par Claude Marois, Montréal, Les Presses de l'université du Québec, 1972, 184 pp.

Probablement seul un géographe est en mesure d'apprécier à sa juste valeur cet Atlas de l'emploi. Pour ma part, je trouve les 184 cartes intéressantes et non dénuées d'un certain esthétisme. II n'aurait certainement pas été inutile, cependant, que l'auteur explique davantage, ou mieux, la façon d'interpréter les ré- 